\title{
The Mechanism of Sertoli-Germ Cell Interaction $^{a}$
}

\author{
M. J. WELSH, M. IRELAND, J. METTS, K. TREINEN, \\ AND G. PITTENGER \\ Department of Anatomy and Cell Biology \\ The University of Michigan Medical School \\ Ann Arbor, Michigan 48109
}

It has long been hypothesized that Sertoli cell (SC) function is affected by the neighboring germ cells (GC). Using two-dimensional gel electrophoresis followed by autoradiography of the gels (2-D autoradiography), we observe that GC or GCconditioned medium (GC-medium) causes rapid, dose-dependent, cell-specific changes in ${ }^{32} \mathrm{P}$ incorporation into proteins of cultured $\mathrm{SC}$. Our results indicate that GC interact with SC by way of the phosphatidylinositol (PI) pathway.

For these studies, SC were isolated from young rats and were cultured for three days. ' SC cultures were completely free of GC. SC were incubated with ${ }^{32} \mathrm{P}$ for $45 \mathrm{~min}\left({ }^{32} \mathrm{P}\right.$ equilibrates with SC ATP in $20 \mathrm{~min}$ ). After various short-term treatments, SC proteins were prepared and subjected to 2-D autoradiography.'

Several proteins showed increased ${ }^{32} \mathrm{P}$ labeling after dibutyryl cyclic AMP (db cAMP) treatment and were also affected by FSH treatment. ${ }^{1,2}$ These proteins may be substrates for cAMP-dependent protein kinase (FIG. 1). Treating SC with ionophore A23187 increased ${ }^{32} \mathrm{P}$ labeling of a $M_{\mathrm{r}} 26 \mathrm{~K}$, pI 5.6 protein (p26), but only if calcium was present in the medium. p26 may be a substrate for calcium/ calmodulin-dependent protein kinase (FIG. 2). Treating SC with ionophore plus 12-O-tetradecanoylphorbol-13-acetate (TPA) caused increased phosphorylation of a $M_{\mathrm{r}} 14 \mathrm{~K}$, pI 4.9 protein (p14). p14 may be a substrate for calcium/phospholipid-dependent protein kinase (FIG. 2). Neither p26 nor p14 was affected by db cAMP.

p26 and p14 also showed increased ${ }^{32} \mathrm{P}$ labeling after treatment with GC or GC medium $^{3}$ (FiG. 2). After one min exposure to GC, labeling of p26 doubled, and by 5 min labeling increased 5 -fold. The response was dose-dependent. p26 seems to be developmentally expressed in SC; it was observed in SC from 18-day-old or older rats, but was not evident in SC from 14-day-old rats. A GC effect on a p26 in the Sertoli cell-derived TR-ST cell line was seen. No GC effect was observed in Chinese hamster ovary (CHO) cells, and both GC and CHO cells lacked p26.

Increased labeling in response to GC or GC medium was also observed in p14. p14 was present in SC from all ages of rats examined and in GC, but a protein with identical 2-D gel mobility was lacking in TR-ST or CHO cells. Current studies in our laboratory include characterization of the GC factor that affects SC, purification of p14 and p26, and elucidation of the PI metabolic pathway in SC.

Our results support two hypotheses: Sertoli cells and germ cells interact by way of the PI pathway, and Sertoli cells have multiple response pathways. Some stimuli may activate one pathway whereas other stimuli may affect distinctly different events.

a This work was supported in part by NIH Grant HD17121 to M. J. Welsh. 


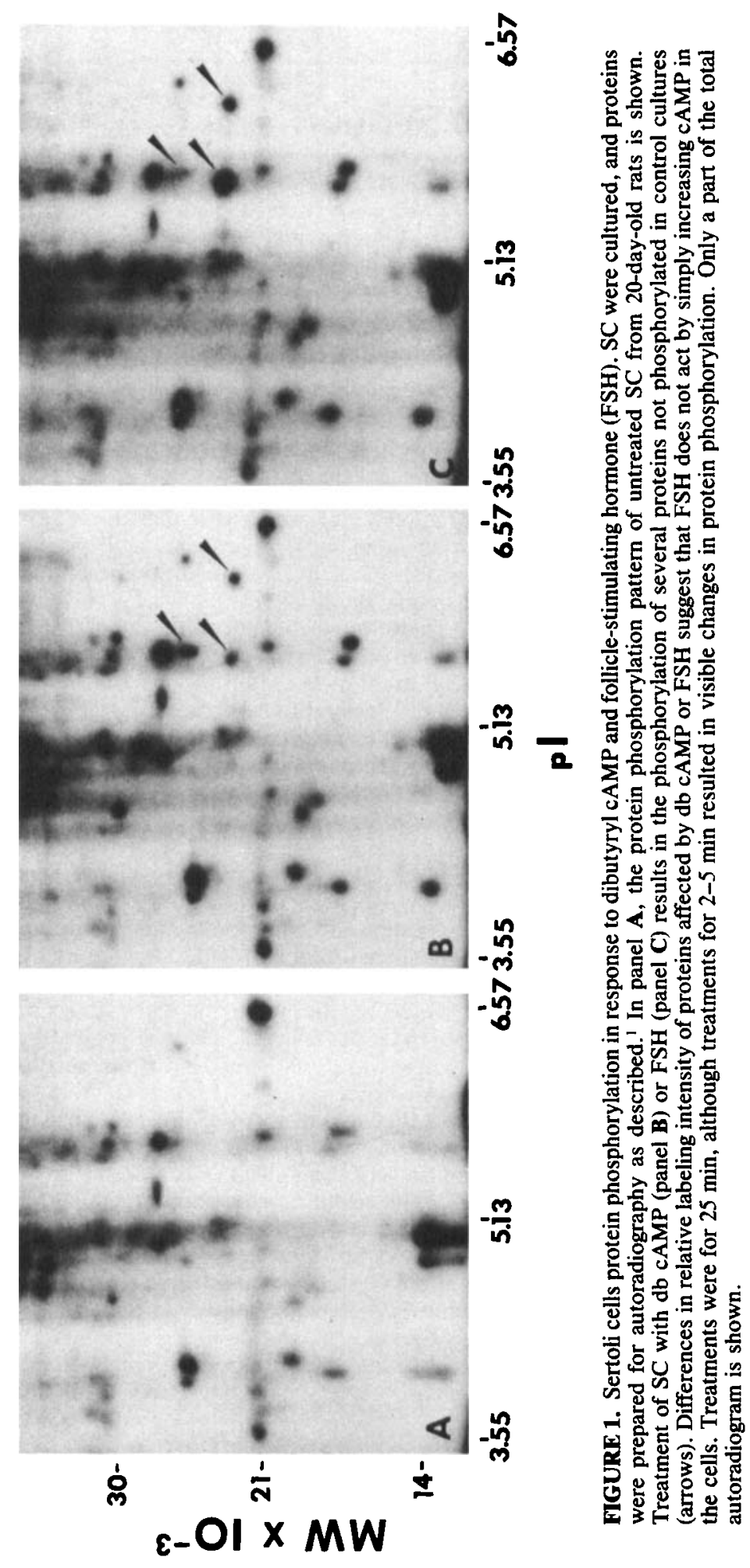




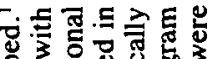

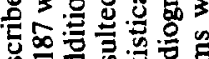

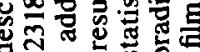

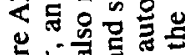
更

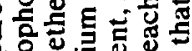

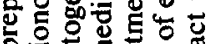

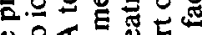

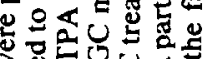

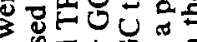
웅뭉을 즘

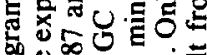

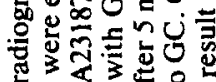

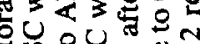

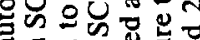

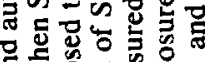

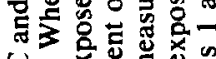
论

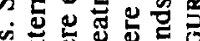

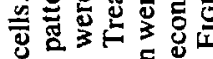
.00் 0 .

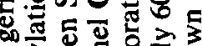

$\infty$

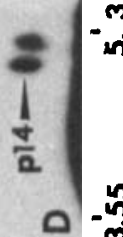

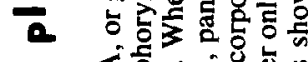

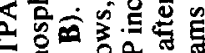
드을 $\infty$.

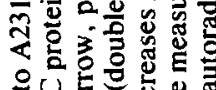

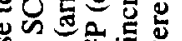

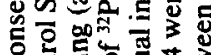

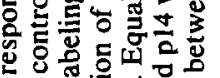

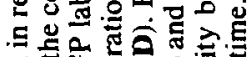
家 范获

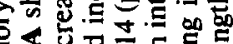

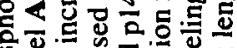

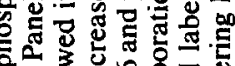

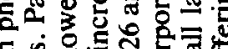

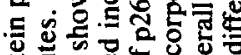

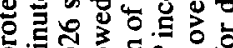

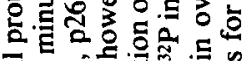

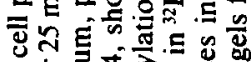

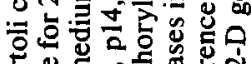

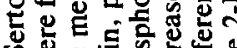
के 슬 넌

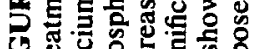

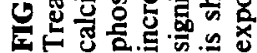




\section{REFERENCES}

1. IRELAND, M. E., B. B. Rosenblum \& M. J. Welsh. 1986. 2-Dimensional gel analysis of Sertoli cell protein phosphorylation: effect of short term exposure to follicle stimulating hormone. Endocrinology 118: 526-532.

2. Ireland, M. E., B. B. Rosenblum \& M. J. Welsh. 2-Dimensional gel analysis of Sertoli cell protein phosphorylation: effect of short term exposure to dibutyryl cyclic AMP, calcium ionophore A23187 and the phorbol ester TPA. Submitted to Biology of Reproduction.

3. IRELAND, M. E. \& M. J. Welsh. 1987. Germ cell stimulation of Sertoli cell protein phosphorylation. Endocrinology. 120: 1317-1326. 